\title{
Axiomatization of Models for Intermediate Logics Constructed with Boolean Models by Piling Up
}

\author{
By \\ Tsutomu Hosor* and Hiroakira ONO
}

In [2] has been given a constructive method for the axiomatization of finite intermediate models. But the application of which, though constructive by nature, to even rather small finite models is already beyond man or computer's capacity. Besides, even if applied, obtained axioms are usually too complicated to be dealt with. So it is often the case, when we study the lattice structure of the intermediate logics, that we feel feeble with the lack of knowledge of axiomatizations of models and that it is wanted to have at hand a simple and handy axiomatization for such an often discussed model as $\boldsymbol{S}_{m} \uparrow \boldsymbol{S}_{1}^{n}$.

For the case of infinite models, Jankov [5] has given an example of models which are not finitely axiomatizable. But we should try, we think, to contrive a way of axiomatization for infinite models, as far as axiomatizable, for the facility of the study of logics.

Here we give an axiomatization for models, possibly infinite, of the form $\boldsymbol{S}_{1}^{n_{1}} \uparrow \boldsymbol{S}_{1}^{n_{2}} \uparrow \cdots \uparrow \boldsymbol{S}_{1}^{n_{k}}$, that is, models constructed with Boolean models by pile operation (piling). We don't assert that this axiomatization is important by itself. But, as mentioned above, these models are often used when discussing intermediate logics and their axiomatization has been hoped for.

On the other hand, for the study of the intermediate logics, we introduced three operations for $\operatorname{logics,} \boldsymbol{M} \cap \boldsymbol{N}, \boldsymbol{M} \cup \boldsymbol{N}$ and $\boldsymbol{M} \uparrow \boldsymbol{N}$. The

Received September 7, 1971.

* Department of Mathematics, Tsuda College, Tokyo 187, Japan. 
former two are defined for logics. Further, if $\boldsymbol{M}$ and $\mathbf{N}$ have been axiomatized, the axiomatic systems for $\boldsymbol{M} \cap \boldsymbol{N}$ and $\boldsymbol{M} \cup \boldsymbol{N}$ are easily obtained. The last operation piling $\boldsymbol{M} \uparrow \boldsymbol{N}$, however, is defined on the basis of model representations of $\boldsymbol{M}$ and $\boldsymbol{N}$, giving different logics for different representations. It provides a difficult open problem to seize the pile operation by the axiomatic method. Our purpose of this paper is to attack this problem. And here we give a first clue for this problem by partly axiomatizing those models mentioned above.

We suppose familiarity with [3], and notations and results in it will be often used without special notices.

\section{§1. Preliminaries}

First, we prepare some definitions and lemmas, most of which are borrowed from [3]. Except when mentioned otherwise, we use lower (upper) case Latin letters for propositional variables (for well-formed formulas). Bold upper case letters are preserved for logics. Lower case Greek letters are for values of models. As models for intermediate logics, we only use pseudo-Boolean models, that is, relatively pseudo-complemented lattice with the maximum and the minimum elements. As this is the case, an ordered relation $\geqq$ is already defined for each model, with the designated element as the minimum. We take $1_{M}$ as the minimum (and the sole designated) value of a model $\boldsymbol{M}$ and $\omega_{\boldsymbol{M}}$ as the maximum, both, possibly without the suffix. We use four logical connectives $>$ (implication), $\wedge$ (conjunction), $\vee$ (disjunction) and $\neg$ (negation). The same symbols are used for the corresponding operations in models. Conjunction and disjuction are also used in the forms $\bigwedge_{1 \leqq i \leqq k}$ and $\underset{1 \leqq i \leqq k}{\bigvee_{i}}$ We abbreviate $((a \supset b)$ $\wedge(b \supset a))$ as $a \equiv b$.

By $\boldsymbol{L}$, we mean the intuitionistic propositional logic.

The next definition provides specially named formulas.

Definition 1.1. $Z(a, b)=(a \supset b) \vee(b \supset a)$,

$$
X_{n}=\underset{1 \leqq i<j \leqq n+1}{\bigvee}\left(a_{i} \equiv a_{j}\right)
$$




$$
\begin{aligned}
& \left\{\begin{array}{l}
P_{1}\left(a_{1}\right)=\left(\neg a_{1} \supset a_{1}\right) \supset a_{1}, \\
P_{n+1}\left(a_{1}, \ldots, a_{n+1}\right)=\left(\left(a_{n+1} \supset P_{n}\left(a_{1}, \ldots, a_{n}\right)\right) \supset a_{n+1}\right) \supset a_{n+1}
\end{array}\right. \\
& (n \geqq 1) \text {. }
\end{aligned}
$$

Definition 1.2. An I (C, D, or N) formula is a formula which contains no other logical connectives but implication (conjunction, disjunction, or negation, respectively). An ICN formula is a formula whose logical connectives are implication, conjunction and negation, at the most. Other combinations are defined similarly.

Lemma 1.3. $A \vee B$ and $(A \supset c) \supset((B \supset c) \supset c)$ are interdeducible in $\mathbb{L}$ if $A \vee B$ does not contain the propositional variable $c$.

Proof. This can be easily ascertained.

Corollary 1.4. $Z(a, b)$ and $X_{n}$ are interdeducible in $\mathbb{L}$ with some I formulas.

For the definitions of $\boldsymbol{M} \cap \boldsymbol{N}, \boldsymbol{M} \cup \boldsymbol{N}$, and $\boldsymbol{M} \uparrow \mathbb{N}$, we refer to [3]. It should be noticed, that in constructing $\mathbb{M} \uparrow \mathbf{N}$, first we take the sets of values of $\boldsymbol{M}$ and $\boldsymbol{N}$ to be disjoint and we identify $\omega_{M}$ and $1_{\boldsymbol{N}}$. So, when we speak of $\boldsymbol{M}$-part ( $\boldsymbol{N}$-part) of $\boldsymbol{M} \uparrow \boldsymbol{N}$, we mean the set of values of $\boldsymbol{M} \uparrow \boldsymbol{N}$ constructed from those equal or less (greater) than the former $1_{N}$.

Lemma 1.5. If a logic is obtained by adding to $\mathbb{L}$ some (possibly infinite) ICN formulas as axioms, then it has the finite model property.

This lemma is proved in [8] for the case of finite additions. But this can be easily extended as above.

Lemma $1.6([6])$. For any logic M, there exists a set of models $\left\{\mathbb{M}_{\lambda} \mid \lambda \in \Lambda\right\}$ such that $\mathbb{M} \supset \bigcap_{\lambda \in \Lambda}\left(\mathbf{S}_{1} \uparrow \mathbb{M}_{\lambda}\right)$. 
In the following $\S \S$, we deal with only those axioms that are interdeducible in $\boldsymbol{L}$ with some ICN formulas. Thus, all the logics dealt with have the finite model property. By this fact and by 1.6 , we only have to deal with those logics expressed as $\bigcap_{\lambda \in \Lambda}\left(\boldsymbol{S}_{1} \uparrow \boldsymbol{M}_{\lambda}\right)$ where each $\boldsymbol{M}_{\lambda}$ is finite.

Lemma 1.7 ([7]). If a logic is obtained by adding to $\boldsymbol{L}$ some I formulas as axioms and if it has a finite model, then it is separable. (For the definition of separability, see [1].)

Lemma 1.8. Let $\boldsymbol{M}$ be $\boldsymbol{S}_{p} \uparrow \boldsymbol{S}_{1}^{q} \uparrow \boldsymbol{S}_{r}$ and $\delta$ be the value in $\boldsymbol{M}$ corresponding to the 1 of $S_{1}^{q}$-part. Let $\alpha$ and $\beta$ be values in the $S_{1}^{q}$-part. Then (i) the value $((\alpha \supset \beta) \supset \alpha) \supset \alpha$ is either 1 or $\delta$, and (ii) $((\alpha \supset \beta) \supset \alpha) \supset \alpha=\delta$ if and only if $\alpha=\delta$ and $\beta>\alpha$.

Lemma 1.9. Let $\boldsymbol{M}$ be a model of the form $\boldsymbol{S}_{1} \uparrow \boldsymbol{N}$ and $\alpha$ and $\beta$ be values in $M$. Then $Z(\alpha, \beta)=1$ if and only if $\alpha \geqq \beta$ or $\alpha \leqq \beta$.

These are easily ascertained.

\section{§2. Balloon Type}

In this $\S$, we give an axiomatization for the models of the form $\boldsymbol{S}_{m} \uparrow \boldsymbol{S}_{1}^{n}$, which we call as of balloon type by the resemblance of the shape of the models expressed by the Hasse diagram. The case of $m=1$ has been dealt with in $[4]$.

Definition 2.1. $A=Z(a, b) \vee(\neg \neg a) a)$.

Corollary 2.2. For any $m$ and $n, \quad S_{m} \uparrow S_{1}^{n} \ni A$.

Proof. Suppose that the values $\alpha$ and $\beta$ are incomparable, that is, $Z(\alpha, \beta) \neq 1$. Then $\neg \neg \alpha=\alpha$, since $\alpha$ is a non-minimal value belonging to the $S_{1}^{n}$-part of the model.

Lemma 2.3. Let $\boldsymbol{M}$ be a finite model of the form $\boldsymbol{S}_{m} \uparrow \boldsymbol{N}$ containing 
$A$ where $\boldsymbol{N}$ is not of the form $\boldsymbol{S}_{1} \uparrow \boldsymbol{N}^{\prime}$. Then there exists an integer $k \geqq 2$ such that $\mathbf{N}$ is isomorphic to $\mathbf{S}_{1}^{k}$ as ordered sets.

Proof. Let $\mathrm{W}$ be the set of values $\{\alpha \mid \neg \alpha \neq 1, \omega\}$. First we prove that $\mathrm{W} \neq \phi$. Suppose otherwise. Then, for any value $\alpha, \neg \alpha=1$ or $\omega$. This means that $\boldsymbol{N}$ is of the form $\boldsymbol{N}^{\prime} \uparrow \boldsymbol{S}_{1}$. Since $\boldsymbol{M}$ is not a linear model, there exists a pair of incomparable values $\alpha$ and $\beta$. Now, $Z(\alpha, \beta) \neq 1$. Since $\boldsymbol{N}$ is of the form $\boldsymbol{N}^{\prime} \uparrow \boldsymbol{S}_{1}, \neg \neg \alpha=1$, yielding $\neg \neg \alpha \supset \alpha=\alpha \neq 1$. Contradiction. Next, let be that $\alpha \in \mathrm{W}$. Then, $\alpha$ and $\neg \alpha$ are incomparable. So, $Z(\alpha, \neg \alpha) \neq 1$. Since $\boldsymbol{M} \ni A, \neg \neg \alpha \supset \alpha$ must be 1 . Hence, if $\alpha \in \mathrm{W}, \neg \neg \alpha \supset \alpha=1$. Now, let $\delta$ be the minimal value of $\boldsymbol{N}$-part of $\boldsymbol{M}$. Then, we can prove that if $\delta<\alpha<\omega, \alpha \in \mathrm{W}$. Suppose that $\alpha \notin \mathrm{W}$. $\neg \alpha$ must be $\omega$. Then $\neg \neg \alpha=1$. Suppose that there exists a value $\beta$ incomparable with $\alpha$. Then $Z(\alpha, \beta) \vee(\neg \neg \alpha) \alpha) \neq 1$. Contradiction. Hence, $\alpha$ is comparable with any value. Therefore, there must be a pair of incomparable values $\beta$ and $\gamma$ between $\delta$ and $\alpha$. Then, again, $Z(\beta, \gamma) \vee(\neg \neg \beta \supset \beta) \neq 1$. Contradiction. Thus, $\mathrm{W} \cup\{\delta, \omega\}$ is isomorphic to some finite Boolean model as ordered sets.

Theorem 2.4. $L+A \supset \subset \boldsymbol{S}_{\omega} \cap \bigcap_{m, n<\omega}\left(\boldsymbol{S}_{m} \uparrow \boldsymbol{S}_{1}^{n}\right) \supset \subset \boldsymbol{S}_{\omega} \uparrow \boldsymbol{S}_{1}^{\omega}$

Proof. We prove only the first relation. By 2.2, $\bigcap_{m, n<\omega}\left(\boldsymbol{S}_{m} \uparrow \boldsymbol{S}_{1}^{n}\right)>\boldsymbol{L}+A$ and $\boldsymbol{S}_{\omega}>\boldsymbol{L}+A$. By 1.5 , there exists a set of finite models $\left\{\boldsymbol{M}_{\lambda} \mid \lambda \in \Lambda\right\}$ such that $\boldsymbol{L}+A \supset \bigcap_{\lambda \in A}\left(\boldsymbol{S}_{1} \uparrow \boldsymbol{M}_{\lambda}\right)$. By 2.3, each $\boldsymbol{S}_{1} \uparrow \boldsymbol{M}_{\lambda}$ is of the form $\boldsymbol{S}_{m} \uparrow \boldsymbol{S}_{1}^{n}$ for some $m$ and $n$ or $\boldsymbol{S}_{k}$ for some $k(1 \leqq k \leqq \omega)$. Hence, $\boldsymbol{L}+A \supset \boldsymbol{S}_{\omega} \cap \bigcap_{m, n<\omega}\left(\mathbf{S}_{m} \uparrow \boldsymbol{S}_{1}^{n}\right)$.

Corollary 2.5. $S_{n} \uparrow S_{1}^{\omega} \supset \subset L+A+P_{n+1}$,

$$
\boldsymbol{S}_{n} \uparrow \boldsymbol{S}_{1}^{k} \supset \mathcal{L}+A+P_{n+1}+X_{\left(2^{k}+n\right)} \text {. }
$$

Corollary 2.6. $S_{n} \uparrow S_{1}^{k}$ is separable.

In most of the succeeding $\S \S$, proofs go almost similarly as above. 
So, details will be often omitted.

\section{§3. Phi Type}

In this $\S$, we give axiomatization for the models of the form $\boldsymbol{S}_{m} \uparrow \boldsymbol{S}_{1}^{n} \uparrow \boldsymbol{S}_{1}$, which we call as of $\phi$ type by the analogy of the shapes of their Hasse diagrams.

Definition 3.1. $B=Z(a, b) \vee\left((\neg a \vee \neg \neg a) \wedge P_{2}(c, a)\right)$.

Corollary 3.2. For any $m$ and $n, M=\boldsymbol{S}_{m} \uparrow \boldsymbol{S}_{1}^{n} \uparrow \boldsymbol{S}_{1} \ni B$.

Proof. Suppose $Z(\alpha, \beta) \neq 1$. Then $\alpha$ and $\beta$ belong to $S_{1}^{n}$-part of $\mathbb{M}$ and they are incomparable. In this case, $\neg \alpha=\omega$, yielding $\neg \alpha \vee \neg \neg \alpha$ $=1$. Suppose $\gamma=\omega$, then $\neg \neg \gamma=1$, yielding $(\neg \gamma \supset \gamma) \supset \gamma=1$, that is, $P_{2}(\gamma, \alpha)=1$. Suppose $\gamma<\omega$, then $\neg \gamma=\omega$, yielding $(\neg \gamma \supset \gamma)>\gamma=\gamma$. If $\gamma$ belongs to $\mathbb{S}_{1}^{n}$-part, then $P_{2}(\gamma, \alpha)=((\alpha \supset \gamma) \supset \alpha) \supset \alpha=1$ by 1.8 . If $\gamma$ belongs to $S_{m}$-part, obviously $P_{2}(\gamma, \alpha)=((\alpha \supset \gamma) \supset \alpha) \supset \alpha=1$.

Lemma 3.3. If a finite model $\mathbf{S}_{1} \uparrow \mathbb{N}$ contains $B$, then there exists $\mathbb{N}^{\prime}$ such that $\mathbb{N}=\mathbb{N}^{\prime} \uparrow \mathfrak{S}_{1}$.

Proof. Suppose otherwise. Then there must exist a value $\alpha$ incomparable with $\neg \alpha$. Then, $Z(\alpha, \neg \alpha) \neq 1$ and $\neg \alpha \vee \neg \neg \alpha \neq 1$, contradiction.

Lemma 3.4. Let be a finite model of the form $\mathbf{S}_{p} \uparrow \mathbb{N} \uparrow \mathbf{S}_{p}$ containing $B$ where $N$ is neither of the form $S_{1} \uparrow \mathbb{N}^{\prime}$ nor $\mathbb{N}^{\prime \prime} \uparrow S_{1}$. Then $q=1$ and there exists an integer $k \geqq 2$ such that $\mathbb{N}$ is isomorphic to $\boldsymbol{S}_{1}^{k}$ as ordered sets.

Proof. First we prove that $q=1$. Suppose that $q \geqq 2$. Let $\gamma$ be the next maximum value, that is, for any $\delta \neq \gamma, \omega, \delta<\gamma$. This $\gamma$ is not the maximum value of $\mathbb{N}$-part in $\mathbb{M}$. In $\mathbb{N}$-part, we can take a pair of 
incomparable values $\alpha$ and $\beta$, that is, $Z(\alpha, \beta) \neq 1$. By this assignment, $P_{2}(\gamma, \alpha)=\alpha \neq 1$ since $(\neg \gamma \supset \gamma) \supset \gamma=\gamma$. Contradiction. Now, let $\varepsilon$ and $\delta$ be the minimum and the maximum values of $\boldsymbol{N}$-part of $\boldsymbol{M}$ and $\mathrm{W}$ be the set $\{\alpha \mid \varepsilon<\alpha<\delta\}$. Then, we can prove that if $\alpha \in \mathrm{W}$, there exists a value $\beta \in \mathrm{W}$ incomparable with $\alpha$. Suppose otherwise. There exists a pair of incomparable values $\beta$ and $\gamma$ between $\varepsilon$ and $\alpha$. Then $Z(\beta, \gamma) \neq 1$ and $P_{2}(\delta, \beta)=\beta \neq 1$. Contradiction. Next, we prove that $((\alpha \supset \delta) \supset \alpha) \supset \alpha$ $=1$ for any $\alpha \in \mathrm{W}$. Suppose that $((\alpha \supset \delta) \supset \alpha) \supset \alpha \neq 1$. Let $\beta$ be a value incomparable with $\alpha$. Since $Z(\alpha, \beta) \neq 1, P_{2}(\gamma, \alpha)$ must be 1 for any $\gamma$. We take $\delta$ as $\gamma$. Since $(\neg \delta \supset \delta) \supset \delta=\delta, P_{2}(\delta, \alpha)=((\alpha \supset \delta) \supset \alpha) \supset \alpha=1$. Contradiction. Thus we have the lemma.

Theorem 3.5. $\quad L+B \supset \subset S_{\omega} \cap \bigcap_{p, k<\omega}\left(S_{p} \uparrow S_{1}^{k} \uparrow S_{1}\right)$

$$
\supset \subset \boldsymbol{S}_{\omega} \uparrow \boldsymbol{S}_{1}^{\omega} \uparrow \boldsymbol{S}_{1}
$$

Proof. Similarly as 2.4.

Corollary 3.6. $S_{n} \uparrow S_{1}^{\omega} \uparrow S_{1}>C L+B+P_{n+2}$,

$$
\boldsymbol{S}_{n} \uparrow \boldsymbol{S}_{1}^{k} \uparrow \boldsymbol{S}_{1}>C \boldsymbol{L}+B+P_{n+2}+X_{\left(2^{k}+n+1\right)} .
$$

Corollary 3.7. $\boldsymbol{S}_{n} \uparrow \boldsymbol{S}_{1}^{k} \uparrow \boldsymbol{S}_{1}$ is separable.

\section{§4. The Simplest Type}

In this $\S$, we treat the simplest type, that is, the models of the form $\mathbf{S}_{m} \uparrow \boldsymbol{S}_{1}^{2} \uparrow \mathbf{S}_{n}$.

Definition 4.1. $C=Z(a, b) \vee Z(c, d) \vee$

$$
((\neg a \vee \neg \neg a) \wedge(((a \equiv c) \wedge(b \equiv d)) \vee((a \equiv d) \wedge(b \equiv c)))) .
$$

Corollary 4.2. For any $m$ and $n, S_{m} \uparrow S_{1}^{2} \uparrow S_{n} \ni C$.

Lemma 4.3. If a finite model $\boldsymbol{M}=\boldsymbol{S}_{1} \uparrow \boldsymbol{N}$ contains $C$, where $\boldsymbol{N}$ is 
not of the form $\boldsymbol{S}_{k}$, then there exist positive integers $m$ and $n$ such that $\boldsymbol{M}=\boldsymbol{S}_{m} \uparrow \boldsymbol{S}_{1}^{2} \uparrow \boldsymbol{S}_{n}$.

Proof. Suppose that there exist two pairs of values $\alpha, \beta, \gamma$, and $\delta$ such that $\alpha$ and $\beta$ are incomparable and that $\gamma$ and $\delta$ are incomparable. Then, since $Z(\alpha, \beta) \neq 1$ and $Z(\gamma, \delta) \neq 1,\{\alpha, \beta\}=\{\gamma, \delta\}$ by the last part of $C$, that is, there only exists a unique pair of values that are incomparable. Since $\neg \alpha \vee \neg \neg \alpha=1$ for this $\alpha, \neg \alpha$ must be $\omega$, that is, $N$ is of the form $\boldsymbol{N}^{\prime} \uparrow \boldsymbol{S}_{1}$. So, we have that $\boldsymbol{M}$ is of the form $\boldsymbol{S}_{m} \uparrow \boldsymbol{S}_{1}^{2} \uparrow \boldsymbol{S}_{n}$ for some $m$ and $n$.

Theorem 4.4. $L+C \supset \subset \boldsymbol{S}_{\omega} \cap \bigcap_{m, n<\omega}\left(\boldsymbol{S}_{m} \uparrow \boldsymbol{S}_{1}^{2} \uparrow \boldsymbol{S}_{n}\right)$

$$
\supset \subset \boldsymbol{S}_{\omega} \uparrow \boldsymbol{S}_{1}^{2} \uparrow \boldsymbol{S}_{\omega}
$$

Proof. Similarly as 2.4 .

Corollary 4.5. $\boldsymbol{L}+C+P_{k+1}>C L+C+X_{k+4}$

$$
\supset \bigcap_{m+n=k}\left(\boldsymbol{S}_{m} \uparrow \boldsymbol{S}_{1}^{2} \uparrow \boldsymbol{S}_{n}\right) .
$$

Now we list up similar results.

Definition 4.6. $C_{n}=Z(a, b) \vee Z(c, d) \vee((\neg a \vee \neg \neg a) \wedge$

$$
\begin{aligned}
& (((a \equiv c) \wedge(b \equiv d)) \vee((a \equiv d) \wedge(b \equiv c))) \wedge \\
& \left.P_{n+1}\left(a_{1}, \ldots, a_{n}, a\right)\right) . \quad(n=1,2, \ldots)
\end{aligned}
$$

Theorem 4.7. $L+C_{n} \supset \subset S_{\omega} \cap \bigcap_{\substack{m<\omega \\ k \leqq n}}\left(S_{m} \uparrow S_{1}^{2} \uparrow S_{k}\right)$

$$
\supset \subset \boldsymbol{S}_{\omega} \uparrow \boldsymbol{S}_{1}^{2} \uparrow \boldsymbol{S}_{n}
$$

Definition 4.8. $C_{m}^{\prime}=Z(a, b) \vee Z(c, d) \vee((\neg a \vee \neg \neg a) \wedge$

$$
(((a \equiv c) \wedge(b \equiv d)) \vee((a \equiv d) \wedge(b \equiv c))) \wedge
$$




$$
\left.F_{m+2}\left(a, a_{1}, \ldots, a_{m+1}\right)\right) . \quad(m=1,2, \ldots)
$$

Theorem 4.9. $\quad \boldsymbol{L}+C_{m}^{\prime} \supset \subset \mathbf{S}_{\boldsymbol{\omega}} \cap \bigcap_{\substack{k \leq m \\ n<\omega}}\left(\mathbf{S}_{k} \uparrow \boldsymbol{S}_{1}^{2} \uparrow \mathbf{S}_{n}\right)$

$$
\supset \subset \boldsymbol{S}_{m} \uparrow \boldsymbol{S}_{1}^{2} \uparrow \boldsymbol{S}_{\omega} \text {. }
$$

Definition 4.10. $C_{m, n}=Z(a, b) \vee Z(c, d) \vee((\neg a \vee \neg \neg a) \wedge$

$$
\begin{gathered}
(((a \equiv c) \wedge(b \equiv d)) \vee((a \equiv d) \wedge((b \equiv c))) \wedge \\
\left.P_{m+2}\left(a, a_{1}, \ldots, a_{m+1}\right) \wedge P_{n+1}\left(b_{1}, \ldots, b_{n}, a\right)\right) . \\
(m=1,2, \ldots ; n=1,2, \ldots)
\end{gathered}
$$

Theorem 4.11. $L+C_{m, n} \supset \subset \boldsymbol{S}_{\omega} \cap\left(\boldsymbol{S}_{m} \uparrow \boldsymbol{S}_{1}^{2} \uparrow S_{n}\right)$.

Corollary 4.12. $S_{m} \uparrow S_{1}^{2} \uparrow S_{n} \supset \subset \boldsymbol{L}+C_{m, n}+P_{m+n+1}$

$$
\begin{aligned}
& \supset \subset \mathrm{L}+C_{n}+P_{m+n+1} \\
& \supset \subset \boldsymbol{L}+C_{m}^{\prime}+P_{m+n+1} .
\end{aligned}
$$

Corollary 4.13. $S_{m} \uparrow S_{1}^{2} \uparrow S_{n}$ is separable.

\section{§5. A Mixed Type}

In this §, we deal with models of the form $\boldsymbol{S}_{p} \uparrow \boldsymbol{S}_{1}^{2} \uparrow \boldsymbol{S}_{q} \uparrow \boldsymbol{S}_{1}^{r}$, mixed of the balloon type and the simplest type. We only give the results, since they can be proved easily by the analogy of the preceding §§.

Definition 5.1. $\left.C_{\omega, \omega}^{*}=C \vee(\neg \neg a) a\right) \vee(\neg \neg c \supset c)$.

Theorem 5.2. $L+C_{\omega, \omega}^{*} \supset \subset S_{\omega} \cap \bigcap_{p, q, r<\omega}\left(S_{p} \uparrow S_{1}^{2} \uparrow S_{q} \uparrow S_{1}^{r}\right)$

$$
\supset \subset \boldsymbol{S}_{\omega} \uparrow \boldsymbol{S}_{1}^{2} \uparrow S_{\omega} \uparrow \boldsymbol{S}_{1}^{\omega}
$$

Definition 5.3. $\left.\left.C_{\omega, n}^{*}=C_{n} \vee(\neg \neg a) a\right) \vee(\neg \neg c) c\right)$. 
Theorem 5.4. $L+C_{\omega, n+1}^{*} \supset \subset S_{\omega} \cap \bigcap_{\substack{p, r<\omega \\ q \leqq n}}\left(S_{p} \uparrow S_{1}^{2} \uparrow S_{q} \uparrow S_{1}^{r}\right)$

$$
\supset \subset S_{\omega} \uparrow S_{1}^{2} \uparrow S_{n} \uparrow S_{1}^{\omega}
$$

Definition 5.5. $\left.\left.C_{m, \omega}^{*}=C_{m}^{\prime} \vee(\neg \neg a) a\right) \vee(\neg \neg c) c\right)$

Theorem 5.6. $L+C_{m, \omega}^{*} \supset \subset \boldsymbol{S}_{\omega} \cap \bigcap_{\substack{p \leqq m \\ q, r<\omega}}\left(\boldsymbol{S}_{p} \uparrow \boldsymbol{S}_{1}^{2} \uparrow \boldsymbol{S}_{q} \uparrow \boldsymbol{S}_{1}^{r}\right)$

$$
\supset \subset S_{m} \uparrow S_{1}^{2} \uparrow S_{\omega} \uparrow S_{1}^{\omega}
$$

Definition 5.7. $\left.C_{m, n}^{*}=C_{m, n} \vee(\neg \neg a \supset a) \vee(\neg \neg c) c\right)$.

Theorem 5.8. $L+C_{m, n+1}^{*} \supset \subset S_{\omega} \cap \bigcap_{\substack{p \leqq m \\ q \leqq n}}\left(\boldsymbol{S}_{p} \uparrow \boldsymbol{S}_{1}^{2} \uparrow \boldsymbol{S}_{q} \uparrow \boldsymbol{S}_{1}^{r}\right)$

$$
\supset \subset \boldsymbol{S}_{\omega} \cap\left(\boldsymbol{S}_{m} \uparrow \boldsymbol{S}_{1}^{2} \uparrow \boldsymbol{S}_{n} \uparrow \boldsymbol{S}_{1}^{\omega}\right) .
$$

Corollary 5.9. $S_{m} \uparrow S_{1}^{2} \uparrow S_{n} \uparrow S_{1}^{\omega} \supset \subset L+C_{m, n+1}^{*}+P_{m+n+2}$

$$
\begin{aligned}
& \supset \subset \mathbb{L}+C_{\omega, n+1}^{*}+P_{m+n+2} \\
& \supset \subset \mathbb{L}+C_{m, \omega}^{*}+P_{m+n+2} .
\end{aligned}
$$

Corollary 5.10. $S_{m} \uparrow S_{1}^{2} \uparrow S_{n} \uparrow S_{1}^{k} \supset \subset L+C_{m, n+1}^{*}+P_{m+n+2}$

$$
+X_{\left(2^{k}+m+n+1\right)}
$$

\section{References}

[1] Hosoi, T., The separation theorem on the classical system, J. Fac. Sci. Univ. Tokyo, Sect. I, 12 (1966), 223-230.

[2] - On the axiomatic method and the algebraic method for dealing with propositional logics, Ibid. 14 (1967), 131-169.

[3] - On intermediate logics I, Ibid. 14 (1967), 293-312.

[4] Hosoi, T. and H. Ono, The intermediate logics on the second slice, Ibid. 17 (1970), 457-461.

[5] Jankov, V. A., Constructing a sequence of strongly independent superintuitionistic propositional calculi, Soviet Math. Dokl. 9 (1968), 806-807.

[6] McKay, C. G., On finite logics, Indag. Math. 29 (1967), 363-365. 
[7] $ـ$ The non-separability of a certain finite extension of Heyting's propositional logic, Ibid. 30 (1968), 312-315.

[8] , The decidability of certain intermediate propositional logics, J. Symbolic Logic, 33 (1968), 258-264. 
